\title{
Las creencias parentales en el proceso de crianza y sus relaciones con el comportamiento adolescente
}

\author{
Miguel Morales-Castillo $\oplus^{*}$ \\ Universidad Nacional de Colombia. Bogotá, Colombia
}

\begin{abstract}
Resumen: Las creencias parentales están integradas estructuralmente en la relación entre padres e hijos y pueden ser consideradas como contribuciones para comprender el desarrollo adolescente. El presente trabajo busca analizar los vínculos entre las creencias parentales y el comportamiento adolescente, para ello se desarrolló una revisión bibliográfica en seis bases de datos, que incluyó 53 estudios en donde se dimensiona la influencia de las creencias parentales en la crianza con adolescentes. En el análisis de datos, se destacaron como "creencias parentales" los siguientes conceptos: las creencias generales sobre los atributos y las experiencias humanas, las expectativas parentales, las percepciones sobre las capacidades de los hijos, los valores y metas parentales, la autoeficacia parental y las creencias sobre el control de las situaciones. Se concluye que las creencias parentales son afectadas por los atributos y las experiencias de los padres, lo que influye en un efecto de cascada sobre el comportamiento adolescente.
\end{abstract}

Palabras clave: creencias parentales, crianza, adolescencia, prácticas de crianza.

\section{Introducción}

La crianza es la orientación de esfuerzos parentales que influyen en el bienestar, el aprendizaje y el cultivo de valores de los hijos, los cuales han sido estudiados en términos de las prácticas de crianza como acciones parentales que inciden en el desarrollo de las niñas, los niños y adolescentes (NNA), las pautas de crianza, o normas que buscan regular el comportamiento de NNA, los estilos, o climas actitudinales en las relaciones entre padres e hijos, así como las creencias parentales o ideas de los padres implicadas en el proceso de crianza (Aguirre-Dávila, 2015; Power, 2013).

Las creencias son juicios subjetivos sobre la relación probable entre objetos, eventos, valores, conceptos y/o atributos, los cuales son obtenidos de la experiencia (Fishbein \& Ajzen, 1975). Dicha relación expresa una representación ontológica del mundo que facilita enfrentar situaciones cotidianas con la provisión de criterios para actuar y evaluar actuaciones (Halligan, 2007), convirtiéndose en convicciones primarias acerca de los eventos, la agencia y los atributos que las personas utilizan y aceptan (Dweck, 2012). Las creencias actúan como filtros, marcos y guías, que intervienen en la cotidianidad como factores que valoran diferencialmente la información disponible (Sharot \& Garrett, 2016) y permiten resolver las inconsistencias al restaurar el contexto de significación (Hall \& Raimi, 2018).

Se han utilizado varios términos para hacer referencia a las ideas de los padres en la crianza

* Dirección para correspondencia: jmmoralesc@unal.edu.co
(Sigel, McGillicuddy-De Lisi, \& Goodnow, 1992); sin embargo, el concepto de creencia resulta adecuado en este contexto, ya que resalta el nivel intuitivo de elaboración personal en situaciones cotidianas y admite el grado probabilístico de relación entre entidades, eventos y atributos.

Entre los tipos de creencias parentales analizados se encuentran las creencias generales sobre los atributos y las experiencias humanas (Dweck, 2012), las expectativas parentales como creencias sobre la posibilidad que tienen los hijos de conseguir determinados resultados (Davis-Kean, 2005), y las percepciones sobre las capacidades de los hijos en tanto creencias sobre el nivel de competencia de los adolescentes en dominios (Simpkins, Fredricks, \& Eccles, 2015). Además, los valores parentales incluyen ideas acerca del nivel de importancia y utilidad de diferentes aspectos (Gniewosz \& Noack, 2012a), mientras que las metas abarcan los objetivos que persiguen los padres cuando interactúan con sus hijos (Gonida, Voulala, \& Kiosseoglou, 2009), y, por último, la autoeficacia parental se refiere al conjunto de creencias sobre la capacidad que los padres perciben de ser eficientes en la crianza (Shumow \& Lomax, 2001).

Por otro lado, la adolescencia es un periodo crítico en la vida de los hijos por los cambios manifiestos en diferentes esferas, una etapa entre los 11 y 21 años segmentada en temprana, media y tardía (Blum, Astone, Decker, \& Mouli, 2014). El comportamiento de los hijos responde a una configuración de factores personales y contextuales, en la cual la relación con los padres puede resultar valiosa para comprender la adolescencia, si bien se experimentan modificaciones que redefinen el vínculo (Pomerantz, Moorman, \& Litwack, 2007). 
En este contexto, emergen interrogantes acerca de la naturaleza de las creencias parentales y sus asociaciones con otras variables, así como sus implicaciones para el desarrollo de NNA (Schaffer, 2006). Trabajos anteriores han propuesto de manera general la relación entre las creencias parentales y el comportamiento de los hijos (Sigel \& McGillicuddy-De Lisi, 2002), mientras que aparece disperso el análisis específico cuando los hijos se encuentran en la adolescencia. Así mismo, revisiones previas han incluido a las creencias como parte del repertorio parental al interactuar con los hijos (MoralesCastillo \& Aguirre-Dávila, 2018), sin profundizar en la particularidad de las creencias parentales. Con estos referentes, el presente trabajo plantea la pregunta: ¿Qué relación existe entre las creencias parentales implicadas en la crianza y las tendencias comportamentales de los hijos en la adolescencia?

\section{Metodología}

En el segundo semestre de 2018, fueron consultadas las bases Taylor and Francis, ScienceDirect, PsycArticles, $S A G E$, EBSCO-Academic y Redalyc, buscando artículos identificados con las palabras clave "parental belief" y "adolescent", lo que permitía circunscribir el campo de estudio a trabajos enfocados en las creencias parentales con hijos adolescentes.

Los documentos debían cumplir con los siguientes criterios de inclusión: (1) Estudios con datos empíricos analizados cuantitativamente que dimensionaban la relación entre las creencias parentales y el comportamiento adolescente; y (2) Artículos revisados por pares. Se consideraron como criterios de exclusión: (1) Estudios en idiomas diferentes al inglés, español, portugués y francés; y (2) Editoriales, comunicaciones, revisiones. análisis:

Se establecieron las siguientes categorías de

- Año: Año de publicación del artículo.

- Origen: Región de origen de los participantes.

- Muestra: Tamaño de la muestra.

- Tipo de estudio: Carácter transversal (T) o longitudinal (L) del diseño.

- Método: Estrategia estadística utilizada para el análisis de datos.

- Creencia parental: Concepto utilizado que corresponde a las creencias parentales.

- Relación entre las variables: Tipo de relación entre las creencias parentales y el comportamiento adolescente.

- Variable influenciada: Aspecto del comportamiento adolescente analizado.

\section{Resultados}

Se identificaron, en total, durante la revisión 838 documentos, de los cuales se seleccionaron finalmente 53, luego de tres rondas (descarte por cumplimiento de criterios al revisar el título, el resumen y el texto completo), prescindiéndose de 793 documentos que no cumplían con los criterios de inclusión, como resume la Figura 1.

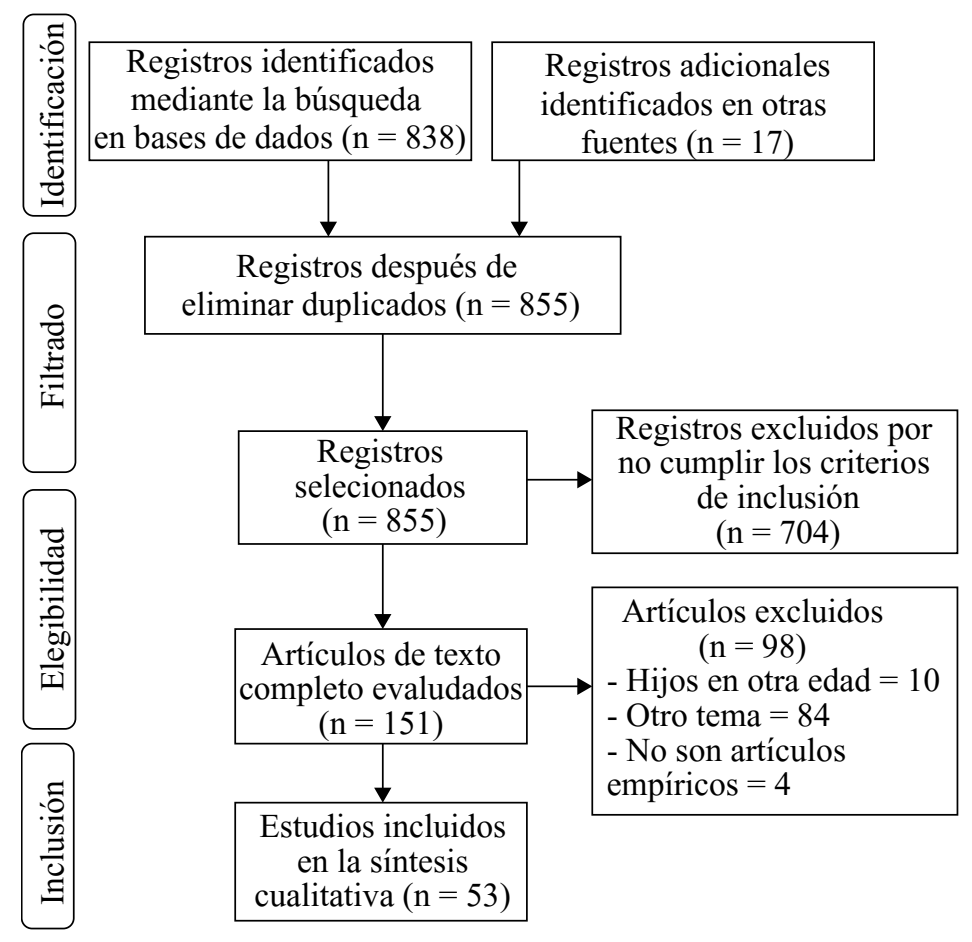

Figura 1. Diagrama de flujo sobre el tratamiento de la información recolectada

Fuente: Elaboración propia. 
Los artículos finalmente seleccionados se distribuyeron entre los años 1982 y 2018 , con un $67,9 \%$ de los artículos concentrados en el rango 2011-2018, como se aprecia en la Figura 2. La mayoría de los estudios provinieron de Norteamérica, con una concentración en Estados Unidos (61,1\%), seguidos por Europa $(18,5 \%)$, Asia $(14,8 \%)$ y Suramérica $(3,7 \%)$, más un trabajo internacional con países de regiones geográficas diferentes (Colombia, Italia y Estados Unidos).

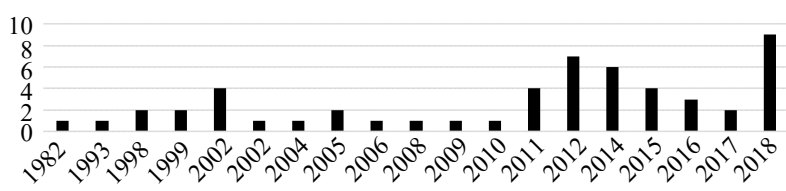

Figura 2. Años cubiertos por los artículos

Fuente: Elaboración propia.

El 55,6\% de los trabajos tuvieron menos de 500 participantes, con un rango entre 28 y 486 casos analizados, mientras que el $24,1 \%$ tuvo entre 500 y 1000 participantes, el 9,3\% entre 1000 y 1500 , y el $11,1 \%$ tuvo más de 1500 , con tres estudios de más de 3 mil participantes. La mayor parte de los artículos analizados fueron transversales con un $68,5 \%$, mientras que los estudios longitudinales constituyeron el 31,5\%; y cabe mencionar que cinco de los diseños longitudinales contaron con muestras mayores a 1000 participantes.

El método estadístico más usado en el análisis de los datos fue el modelo de ecuaciones estructurales $(23,6 \%)$, seguida del análisis de senderos (16,4\%), del análisis de regresión múltiple (12,7\%), del análisis de regresión jerárquico $(10,9 \%)$ y la regresión logística $(7,3 \%)$.

Como recoge la Tabla 1, seis tipos de conceptos fueron incluidos en los estudios recopilados dentro de la categoría "creencias parentales": las creencias sobre los atributos y las experiencias humanas $(22,6 \%)$, las expectativas parentales $(20,8 \%)$, las percepciones sobre las capacidades de los hijos $(18,9 \%)$, los valores y las metas parentales $(18,9 \%)$, la autoeficacia parental $(15,1 \%)$ y las creencias sobre el control de las situaciones $(3,8 \%)$.

Tabla 1. Resumen de estudios

\begin{tabular}{|c|c|c|c|c|c|c|c|}
\hline Referencia & Origen & $\mathrm{N}$ & Tipo & Método & Creencias parentales & Relación & Variable influenciada \\
\hline $\begin{array}{l}\text { Babskie, Powell \& } \\
\text { Metzger (2017) }\end{array}$ & $\begin{array}{l}\text { Estados } \\
\text { Unidos }\end{array}$ & 161 & $\mathrm{~T}$ & Correlación & Autoeficacia & $\mathrm{D}$ & $\begin{array}{c}\text { Problemas de } \\
\text { comportamiento }\end{array}$ \\
\hline $\begin{array}{l}\text { Bleeker \& } \\
\text { Jacobs (2004) }\end{array}$ & $\begin{array}{l}\text { Estados } \\
\text { Unidos }\end{array}$ & 1007 & $\mathrm{~L}$ & $\begin{array}{l}\text { Análisis de } \\
\text { senderos }\end{array}$ & Percepciones & Md & $\begin{array}{c}\text { Autoeficacia en la } \\
\text { selección de una carrera }\end{array}$ \\
\hline $\begin{array}{l}\text { Bobowik, van } \\
\text { Oudenhoven, Basabe, } \\
\text { Telletxea Páez (2011) }\end{array}$ & España & 102 & $\mathrm{~T}$ & $\begin{array}{l}\text { Análisis de } \\
\text { senderos }\end{array}$ & Metas & $\mathrm{D}$ & $\begin{array}{c}\text { Metas, aspiraciones y } \\
\text { deseos de los hijos }\end{array}$ \\
\hline $\begin{array}{l}\text { Bois, Sarrazin, } \\
\text { Brustad, Trouilloud } \\
\& \text { Cury (2002) }\end{array}$ & Francia & 156 & $\mathrm{~L}$ & $\begin{array}{c}\text { Ecuaciones } \\
\text { estructurales }\end{array}$ & Percepciones & $\mathrm{Mr}$ & $\begin{array}{l}\text { Competencia física } \\
\text { percibida }\end{array}$ \\
\hline Butler \& Le (2018) & China & 189 & $\mathrm{~T}$ & ANOVA & Valores & $\mathrm{Md}$ & Aprendizaje del inglés \\
\hline $\begin{array}{l}\text { Calero, Dalley } \\
\text { Fernandez, Davenport- } \\
\text { Dalley, Morote \& } \\
\text { Tatum (2014) }\end{array}$ & $\begin{array}{l}\text { Estados } \\
\text { Unidos }\end{array}$ & 222 & $\mathrm{~T}$ & $\begin{array}{c}\text { Ecuaciones } \\
\text { estructurales }\end{array}$ & Expectativas & $\mathrm{D}$ & Autoconcepto académico \\
\hline $\begin{array}{l}\text { Cederbaum, Guerrero, } \\
\text { Barman-Adhikari } \\
\text { \& Vincent (2015) }\end{array}$ & $\begin{array}{l}\text { Estados } \\
\text { Unidos }\end{array}$ & 176 & $\mathrm{~T}$ & $\begin{array}{l}\text { Regresión } \\
\text { logística }\end{array}$ & $\begin{array}{l}\text { Creencias sobre el } \\
\text { consumo de alcohol }\end{array}$ & $\mathrm{D}$ & Consumo de alcohol \\
\hline $\begin{array}{l}\text { Chang Presson, C. } \\
\text { C., Rose, J. S., \& } \\
\text { Sherman et al. (2015) }\end{array}$ & Taiwán & 2106 & $\mathrm{~T}$ & $\begin{array}{l}\text { Regresión } \\
\text { logística }\end{array}$ & Autoeficacia & Md & Uso de sustancias \\
\hline $\begin{array}{l}\text { Chassin, Presson, } \\
\text { Rose \& Sherman } \\
(1998)\end{array}$ & $\begin{array}{l}\text { Estados } \\
\text { Unidos }\end{array}$ & 192 & $\mathrm{~L}$ & $\begin{array}{c}\text { Ecuaciones } \\
\text { estructurales }\end{array}$ & Creencias sobre fumar & Md & $\begin{array}{l}\text { Creencias sobre fumar, } \\
\text { experiencia fumando }\end{array}$ \\
\hline $\begin{array}{l}\text { Chen \& Faldowski } \\
\text { (2014) }\end{array}$ & China & 698 & $\mathrm{~T}$ & $\begin{array}{l}\text { Análisis de } \\
\text { frecuencia }\end{array}$ & $\begin{array}{c}\text { Creencias parentales } \\
\text { sobre la autoridad }\end{array}$ & $\mathrm{D}$ & $\begin{array}{c}\text { Creencias de los hijos } \\
\text { sobre la legitimidad } \\
\text { de la autoridad }\end{array}$ \\
\hline
\end{tabular}


Tabla 1. Continuación

\begin{tabular}{|c|c|c|c|c|c|c|c|}
\hline Referencia & Origen & $\mathrm{N}$ & Tipo & Método & Creencias parentales & Relación & Variable influenciada \\
\hline Chen \& Fish (2012) & $\begin{array}{l}\text { Estados } \\
\text { Unidos }\end{array}$ & 189 & $\mathrm{~T}$ & $\begin{array}{l}\text { Regresión } \\
\text { jerárquica }\end{array}$ & Autoeficacia & $\mathrm{D}$ & Logro académico \\
\hline Davis-Kean (2005) & $\begin{array}{l}\text { Estados } \\
\text { Unidos }\end{array}$ & 868 & $\mathrm{~T}$ & $\begin{array}{l}\text { Ecuaciones } \\
\text { estructurales }\end{array}$ & Expectativas & $\mathrm{Md}$ & Logro académico \\
\hline Di Giunta et al. (2018) & $\begin{array}{l}\text { Colombia, } \\
\text { Italia, } \\
\text { Estados } \\
\text { Unidos }\end{array}$ & 534 & $\mathrm{~L}$ & $\begin{array}{l}\text { Análisis de } \\
\text { senderos }\end{array}$ & Autoeficacia & Md & $\begin{array}{c}\text { Tendencias internalizantes } \\
\text { y externalizantes }\end{array}$ \\
\hline $\begin{array}{l}\text { Dickens \& } \\
\text { Cornell (1993) }\end{array}$ & $\begin{array}{l}\text { Estados } \\
\text { Unidos }\end{array}$ & 165 & $\mathrm{~T}$ & $\begin{array}{l}\text { Análisis de } \\
\text { senderos }\end{array}$ & Autoconcepto parental & $\mathrm{Md}$ & $\begin{array}{l}\text { Autoconcepto académico } \\
\text { (matemáticas) }\end{array}$ \\
\hline Dielman et al. (1982) & $\begin{array}{l}\text { Estados } \\
\text { Unidos }\end{array}$ & 854 & $\mathrm{~T}$ & $\begin{array}{l}\text { Regresión } \\
\text { múltiple }\end{array}$ & Ideas sobre la salud & $\mathrm{D}$ & $\begin{array}{c}\text { Creencias y } \\
\text { comportamientos } \\
\text { sobre la salud }\end{array}$ \\
\hline Eng (2012) & Camboya & 202 & $\mathrm{~T}$ & $\begin{array}{l}\text { Regresión } \\
\text { por Mínimos } \\
\text { Cuadrados }\end{array}$ & $\begin{array}{l}\text { Predestinación de } \\
\text { los hijos (fatalismo) }\end{array}$ & $\mathrm{D}$ & Logro académico \\
\hline $\begin{array}{l}\text { Fleming, Sznajder, } \\
\text { Nepps \& Boktor } \\
(2018)\end{array}$ & $\begin{array}{l}\text { Estados } \\
\text { Unidos }\end{array}$ & 722 & $\mathrm{~T}$ & Frecuencias & $\begin{array}{l}\text { Creencias sobre } \\
\text { la vacunación }\end{array}$ & $\mathrm{D}$ & $\begin{array}{l}\text { Vacunación contra el } \\
\text { papiloma humano }\end{array}$ \\
\hline $\begin{array}{l}\text { Fredricks \& } \\
\text { Eccles (2002) }\end{array}$ & $\begin{array}{l}\text { Estados } \\
\text { Unidos }\end{array}$ & 882 & $\mathrm{~L}$ & $\begin{array}{l}\text { Modelamiento } \\
\text { linear } \\
\text { jerárquico }\end{array}$ & Expectativas & $\mathrm{D}$ & $\begin{array}{c}\text { Competencia } \\
\text { percibida, importancia } \\
\text { asignada e interés }\end{array}$ \\
\hline $\begin{array}{l}\text { Freed \& Tompson } \\
\text { (2011) }\end{array}$ & $\begin{array}{l}\text { Estados } \\
\text { Unidos }\end{array}$ & 160 & $\mathrm{~L}$ & $\begin{array}{l}\text { Regresión } \\
\text { jerárquica }\end{array}$ & Locus de control & $\mathrm{D}$ & $\begin{array}{c}\text { Comportamientos } \\
\text { externalizantes }\end{array}$ \\
\hline $\begin{array}{l}\text { Gladstone, Häfner, } \\
\text { Turci, Kneißler \& } \\
\text { Muenks (2018) }\end{array}$ & Alemania & 830 & $\mathrm{~T}$ & $\begin{array}{l}\text { Ecuaciones } \\
\text { estructurales }\end{array}$ & Percepciones & Md & $\begin{array}{c}\text { Autoconcepto académico } \\
\text { adolescente }\end{array}$ \\
\hline $\begin{array}{l}\text { Gniewosz \& } \\
\text { Noack (2012a) }\end{array}$ & Alemania & 1014 & $\mathrm{~L}$ & $\begin{array}{l}\text { Ecuaciones } \\
\text { estructurales }\end{array}$ & Valores & $\mathrm{Md}$ & $\begin{array}{l}\text { Importancia dada por los } \\
\text { hijos hacia la educación }\end{array}$ \\
\hline $\begin{array}{l}\text { Gniewosz \& } \\
\text { Noack (2012b) }\end{array}$ & Alemania & 1019 & $\mathrm{~L}$ & $\begin{array}{l}\text { Ecuaciones } \\
\text { estructurales }\end{array}$ & Valores & $\mathrm{Md}$ & $\begin{array}{l}\text { Importancia dada por los } \\
\text { hijos hacia áreas académicas }\end{array}$ \\
\hline Gonida et al. (2009) & Grecia & 271 & $\mathrm{~T}$ & $\begin{array}{l}\text { Análisis de } \\
\text { senderos }\end{array}$ & Metas & $\mathrm{Md}$ & $\begin{array}{l}\text { Metas de logro, } \\
\text { compromiso escolar }\end{array}$ \\
\hline $\begin{array}{l}\text { Gonida, Karabenick, } \\
\text { Makara \& } \\
\text { Hatzikyriakou (2014) }\end{array}$ & Grecia & 712 & $\mathrm{~T}$ & $\begin{array}{l}\text { Análisis de } \\
\text { senderos }\end{array}$ & Metas & $\mathrm{Md}$ & $\begin{array}{l}\text { Actitudes hacia la búsqueda } \\
\text { de ayuda académica }\end{array}$ \\
\hline $\begin{array}{l}\text { Heitzler, Levin, Duke } \\
\& \text { Huhman (2006) }\end{array}$ & $\begin{array}{l}\text { Estados } \\
\text { Unidos }\end{array}$ & 3114 & $\mathrm{~T}$ & $\begin{array}{c}\text { Regresión } \\
\text { logística }\end{array}$ & $\begin{array}{l}\text { Creencias acerca de } \\
\text { la actividad física }\end{array}$ & $\mathrm{D}$ & Actividad física \\
\hline $\begin{array}{l}\text { Her \& Dunsmore } \\
\text { (2011) }\end{array}$ & $\begin{array}{l}\text { Estados } \\
\text { Unidos }\end{array}$ & 60 & $\mathrm{~T}$ & MANOVA & $\begin{array}{l}\text { Creencias acerca } \\
\text { de las emociones }\end{array}$ & $\mathrm{D}$ & Autoesquemas personales \\
\hline $\begin{array}{l}\text { Hofferth \& } \\
\text { Jeong (2016) }\end{array}$ & $\begin{array}{l}\text { Estados } \\
\text { Unidos }\end{array}$ & 441 & $\mathrm{~L}$ & $\begin{array}{c}\text { Regresión } \\
\text { logística }\end{array}$ & Expectativas & $\mathrm{D}$ & $\begin{array}{c}\text { Graduación del colegio, } \\
\text { ingreso a la universidad, } \\
\text { empleo, encarcelamiento, } \\
\text { tener hijos }\end{array}$ \\
\hline $\begin{array}{l}\text { Holmes, Bond \& } \\
\text { Byrne (2008) }\end{array}$ & $\begin{array}{l}\text { Estados } \\
\text { Unidos }\end{array}$ & 52 & $\mathrm{~T}$ & $\begin{array}{l}\text { Regresión } \\
\text { múltiple }\end{array}$ & Creencias epistémicas & $\mathrm{D}$ & $\begin{array}{l}\text { Creencias de los hijos } \\
\text { sobre el conflicto }\end{array}$ \\
\hline $\begin{array}{l}\text { Jensen \& McHale } \\
(2015)\end{array}$ & $\begin{array}{l}\text { Estados } \\
\text { Unidos }\end{array}$ & 388 & $\mathrm{~L}$ & $\begin{array}{l}\text { Análisis de } \\
\text { senderos }\end{array}$ & Percepciones & $\mathrm{D}$ & Logro académico \\
\hline
\end{tabular}


Tabla 1. Continuación

\begin{tabular}{|c|c|c|c|c|c|c|c|}
\hline Referencia & Origen & $\mathrm{N}$ & Tipo & Método & Creencias parentales & Relación & Variable influenciada \\
\hline $\begin{array}{l}\text { Kimiecik \& } \\
\text { Horn (1998) }\end{array}$ & $\begin{array}{l}\text { Estados } \\
\text { Unidos }\end{array}$ & 81 & $\mathrm{~T}$ & $\begin{array}{l}\text { Regresión } \\
\text { múltiple }\end{array}$ & Percepciones & $\mathrm{D}$ & Actividad física de los hijos \\
\hline $\begin{array}{l}\text { Kirk Lewis- } \\
\text { Moss, Nilsen \& } \\
\text { Colvin }(2011)\end{array}$ & $\begin{array}{l}\text { Estados } \\
\text { Unidos }\end{array}$ & 171 & $\mathrm{~T}$ & $\begin{array}{l}\text { Regresión } \\
\text { lineal }\end{array}$ & Expectativas & $\mathrm{D}$ & Aspiraciones educativas \\
\hline Kung \& Lee (2016) & Taiwán & 1286 & $\mathrm{~T}$ & $\begin{array}{l}\text { Ecuaciones } \\
\text { estructurales }\end{array}$ & Expectativas & $\mathrm{D}$ & $\begin{array}{l}\text { Logro académico } \\
\text { en matemáticas }\end{array}$ \\
\hline Lamb \& Crano (2014) & $\begin{array}{l}\text { Estados } \\
\text { Unidos }\end{array}$ & 3131 & $\mathrm{~L}$ & ANCOVA & Expectativas & $\mathrm{D}$ & Consumo de marihuana \\
\hline Leung \& Shek (2014) & China & 432 & $\mathrm{~T}$ & $\begin{array}{l}\text { Análisis de } \\
\text { senderos }\end{array}$ & Expectativas & $\mathrm{D}$ & Motivación académica \\
\hline Leung \& Shek (2018) & China & 275 & $\mathrm{~T}$ & $\begin{array}{l}\text { Regresión } \\
\text { jerárquica }\end{array}$ & Expectativas & $\mathrm{Md}$ & Desarrollo óptimo \\
\hline $\begin{array}{l}\text { Lindner, Hetherington } \\
\text { \& Reiss (1999) }\end{array}$ & $\begin{array}{l}\text { Estados } \\
\text { Unidos }\end{array}$ & 486 & $\mathrm{~T}$ & $\begin{array}{l}\text { Regresión } \\
\text { jerárquica }\end{array}$ & Creencias religiosas & $\mathrm{Md}$ & Responsabilidad social \\
\hline $\begin{array}{l}\text { Lock, Le Grange, } \\
\text { Agras, Fitzpatrick } \\
\text { \& Stainer (2015) }\end{array}$ & $\begin{array}{l}\text { Estados } \\
\text { Unidos }\end{array}$ & 45 & $\mathrm{~L}$ & Prueba T & Autoeficacia & $\mathrm{D}$ & $\begin{array}{l}\text { Habilidades re-alimenticias } \\
\quad \text { (en casos de anorexia) }\end{array}$ \\
\hline Mak (2018) & $\begin{array}{l}\text { Estados } \\
\text { Unidos }\end{array}$ & 3232 & $\mathrm{~L}$ & $\begin{array}{l}\text { Regresión } \\
\text { por mínimos } \\
\text { cuadrados }\end{array}$ & Percepciones & $\mathrm{D}$ & Beber y fumar \\
\hline $\begin{array}{l}\text { Malmberg, Ehrman } \\
\text { \& Lithén }(2005)\end{array}$ & $\begin{array}{l}\text { Estados } \\
\text { Unidos }\end{array}$ & 197 & $\mathrm{~T}$ & $\begin{array}{c}\text { Ecuaciones } \\
\text { estructurales }\end{array}$ & $\begin{array}{l}\text { Creencias parentales } \\
\text { sobre el futuro }\end{array}$ & $\mathrm{Md}$ & Creencias sobre el futuro \\
\hline $\begin{array}{l}\text { McDonald, Deakin } \\
\& \text { Aalborg, (2010) }\end{array}$ & $\begin{array}{l}\text { Estados } \\
\text { Unidos }\end{array}$ & 357 & $\mathrm{~T}$ & $\begin{array}{l}\text { Comparación } \\
\text { de grupos }\end{array}$ & Percepciones & $\mathrm{D}$ & $\begin{array}{l}\text { Ir al colegio en bicicleta } \\
\text { y/o caminando }\end{array}$ \\
\hline $\begin{array}{l}\text { McGillicuddy \& } \\
\text { Eliseo-Arras (2012) }\end{array}$ & $\begin{array}{l}\text { Estados } \\
\text { Unidos }\end{array}$ & 50 & $\mathrm{~T}$ & $\begin{array}{l}\text { Regresión } \\
\text { múltiple }\end{array}$ & Percepciones & $\mathrm{D}$ & Consumo de sustancias \\
\hline $\begin{array}{l}\text { Mcneely, Shew, } \\
\text { Beuhring, Sieving } \\
\& \text { Blum (2002) }\end{array}$ & $\begin{array}{l}\text { Estados } \\
\text { Unidos }\end{array}$ & 2006 & $\mathrm{~L}$ & $\begin{array}{c}\text { Riesgos } \\
\text { proporcionales }\end{array}$ & Valores & $\mathrm{D}$ & $\begin{array}{l}\text { Momento de la primera } \\
\text { relación sexual }\end{array}$ \\
\hline Mesurado et al. (2014) & $\begin{array}{l}\text { Argentina, } \\
\text { Colombia, } \\
\text { España }\end{array}$ & 1552 & $\mathrm{~T}$ & Regresión & Expectativas & $\mathrm{D}$ & Comportamiento prosocial \\
\hline $\begin{array}{l}\text { Okagaki, Hammond } \\
\& \text { Seamon (1999) }\end{array}$ & $\begin{array}{l}\text { Estados } \\
\text { Unidos }\end{array}$ & 94 & $\mathrm{~T}$ & $\begin{array}{l}\text { Regresión } \\
\text { múltiple }\end{array}$ & Valores & Md & Religiosidad de los hijos \\
\hline $\begin{array}{l}\text { Pesu, Aunola, } \\
\text { Viljaranta \& } \\
\text { Hirvonen (2018) }\end{array}$ & Finlandia & 745 & $\mathrm{~L}$ & $\begin{array}{l}\text { Curva de } \\
\text { crecimiento } \\
\text { latente }\end{array}$ & Percepciones & Md & Logro académico \\
\hline $\begin{array}{l}\text { Sanders, Parent, } \\
\text { Forehand, Sullivan } \\
\text { \& Jones (2016) }\end{array}$ & $\begin{array}{l}\text { Estados } \\
\text { Unidos }\end{array}$ & 615 & $\mathrm{~L}$ & $\begin{array}{c}\text { Ecuaciones } \\
\text { estructurales }\end{array}$ & Autoeficacia & Md & $\begin{array}{c}\text { Tiempo frente a pantallas y } \\
\text { problemas internalizantes } \\
\text { y externalizantes }\end{array}$ \\
\hline $\begin{array}{l}\text { Shumow \& } \\
\text { Lomax (2002) }\end{array}$ & $\begin{array}{l}\text { Estados } \\
\text { Unidos }\end{array}$ & 929 & $\mathrm{~T}$ & $\begin{array}{c}\text { Ecuaciones } \\
\text { estructurales }\end{array}$ & Autoeficacia & Md & $\begin{array}{l}\text { Ajuste académico y } \\
\text { socioemocional }\end{array}$ \\
\hline Simpkins et al. (2012) & $\begin{array}{l}\text { Estados } \\
\text { Unidos }\end{array}$ & 723 & $\mathrm{~L}$ & $\begin{array}{c}\text { Ecuaciones } \\
\text { estructurales }\end{array}$ & Percepciones & $\mathrm{Md}$ & $\begin{array}{c}\text { Autoconcepto, } \\
\text { importancia y tiempo } \\
\text { dedicado a actividades, } \\
\text { habilidad de los hijos }\end{array}$ \\
\hline
\end{tabular}


Tabla 1. Continuación

\begin{tabular}{|c|c|c|c|c|c|c|c|}
\hline Referencia & Origen & $\mathrm{N}$ & Tipo & Método & Creencias parentales & Relación & Variable influenciada \\
\hline $\begin{array}{l}\text { Šimunović, Reić } \\
\text { Ercegovac \& } \\
\text { Burušić (2018) }\end{array}$ & Croacia & 1071 & $\mathrm{~T}$ & $\begin{array}{l}\text { Análisis de } \\
\text { senderos }\end{array}$ & Valores & $\mathrm{Md}$ & $\begin{array}{l}\text { Importancia asignada a la } \\
\text { ciencia y la tecnología }\end{array}$ \\
\hline $\begin{array}{l}\text { Sorkkila, Aunola, } \\
\& \text { Ryba (2017) }\end{array}$ & Finlandia & 448 & $\mathrm{~T}$ & $\begin{array}{l}\text { Ecuaciones } \\
\text { estructurales }\end{array}$ & Expectativas & Md & Burnout deportivo y escolar \\
\hline $\begin{array}{l}\text { Valois, Rizzo, Wanick, } \\
\text { Cavalcanti \& Assis } \\
\text { (2012) }\end{array}$ & Brasil & 126 & $\mathrm{~T}$ & Chi-cuadrado & $\begin{array}{l}\text { Creencias sobre el } \\
\text { ejercicio físico }\end{array}$ & $\mathrm{D}$ & Nivel de actividad física \\
\hline Wang \& Wang (2018) & China & 864 & $\mathrm{~T}$ & $\begin{array}{l}\text { Regresión } \\
\text { jerárquica }\end{array}$ & Atribuciones & Md & $\begin{array}{l}\text { Dificultades para la } \\
\text { regulación emocional }\end{array}$ \\
\hline $\begin{array}{l}\text { Webb \& Whitmer } \\
(2003)\end{array}$ & $\begin{array}{l}\text { Estados } \\
\text { Unidos }\end{array}$ & 167 & $\mathrm{~T}$ & Correlaciones & Valores religiosos & $\mathrm{D}$ & $\begin{array}{l}\text { Mantenimiento de } \\
\text { creencias religiosas }\end{array}$ \\
\hline
\end{tabular}

$\mathrm{T}=$ Transversal; $\mathrm{L}=$ Longitudinal; $\mathrm{D}$ = Directa; $\mathrm{Md}=$ Mediada; $\mathrm{Mr}=$ Mediadora.

En primer lugar, en cuanto a las creencias generales, se establece que si los padres consideran que el conocimiento se recibe pasivamente de un experto hay una percepción negativa del conflicto con los hijos, lo que se asocia con mayor frecuencia e intensidad de este (Holmes et al., 2008); así mismo, cuando los padres creen que los hijos necesitan altos niveles de guía emocional y que las emociones son peligrosas, los hijos desarrollan mayor dependencia (Her \& Dunsmore, 2011). Además, los padres que consideran que las tendencias humanas están predestinadas se asocian negativamente con el logro académico adolescente (Eng, 2012).

Otras creencias sobre las experiencias humanas están relacionadas con la salud, las cuales pueden incidir sobre la susceptibilidad de los hijos a considerar las consecuencias de los comportamientos para la salud (Dielman et al., 1982) o sobre la tendencia de los hijos a no estar vacunados (Fleming et al., 2018). De manera complementaria, lo que los padres creen acerca de la actividad física influye en la práctica de actividades físicas de los hijos (Heitzler et al., 2006), aunque las creencias de las madres pueden no asociarse con los niveles de actividad física de los hijos cuando hay riesgo para la salud adolescente (Valois et al., 2012).

Además, si las madres creen que el consumo de alcohol es normal se elevan las probabilidades de abuso en su consumo por parte de las hijas, lo que influye más que las creencias religiosas parentales (Cederbaum, Guerrero, Barman-Adhikari \& Vincent, 2015), así como el efecto de las creencias parentales sobre fumar puede ser mayor, incluyendo así los comportamientos parentales (Chassin et al., 1998). Igualmente, la creencia parental sobre el control social en torno a sus hijos se asocia con la probabilidad de que estos caminen o vayan en bicicleta al colegio (McDonald et al., 2010).

En segundo lugar, las expectativas parentales pueden estar relacionadas con aspectos personales de los padres como el estatus socioeconómico, lo cual influye positivamente en el logro académico de los hijos (Davis-Kean, 2005), de manera que los niveles altos de las expectativas se asocian con las aspiraciones educativas altas de los hijos (Kirk et al., 2011), mediando la relación entre el estatus parental de origen y el éxito de los hijos, lo que sugiere un efecto protector ante situaciones adversas como las que viven los hijos de inmigrantes (Hofferth \& Jeong, 2016).

Las expectativas influencian las creencias de los hijos sobre su futuro mediante la calidad de las interacciones percibida por los hijos (Malmberg et al., 2005), e inciden positivamente en el desarrollo adolescente con la mediación del control parental (Leung \& Shek, 2018), pero también pueden tener un efecto directo positivo en dominios como el académico (Kung \& Lee, 2016). Su incidencia positiva puede mediar la relación entre otras creencias; por ejemplo, el autoconcepto académico de los padres influye en el autoconcepto de los hijos a través de las expectativas parentales (Dickens \& Cornell, 1993).

Las expectativas parentales se relacionan con el autoconcepto académico de los hijos más que las relaciones con pares y el trabajo escolar (Calero et al., 2014), inciden en la motivación de los adolescentes en asociación con la calidad del funcionamiento familiar (Leung \& Shek, 2014), favorecen el comportamiento prosocial de los hijos (Mesurado, et. Al., (2014) y sus logros educativos (Kung \& Lee, 2016), a pesar de los cambios personales durante la adolescencia (Fredricks \& Eccles, 2002). Sin embargo, el efecto protector de las expectativas puede restringirse a un dominio de referencia y no ser generalizable (Sorkkila et al., 2017).

Así mismo, las expectativas negativas se relacionan con el inicio y aumento del consumo adolescente de marihuana y con restricciones en la comunicación entre padres e hijos (Lamb \& Crano, 2014), lo que reduce las oportunidades para acceder a información valiosa sobre los comportamientos de riesgo.

En tercer lugar, las percepciones sobre las capacidades de los hijos influyen en el comportamiento parental, en las creencias y en los comportamientos de los 
hijos (Simpkins et al., 2012), dado que las percepciones maternales favorables sobre la competencia física del adolescente hacen más probable que este se perciba como físicamente competente (Bois, Sarrazin et al., 2002) y los padres que creen en la competencia de sus hijos en actividades físicas tienen hijos más activos físicamente (Kimiecik \& Horn, 1998). Las percepciones parentales sobre las diferencias entre sus hijos pueden predecir las diferencias reales en los resultados de estos (Jensen \& McHale, 2015). Además, los padres de adolescentes hombres son más propensos a creer que sus hijos son buenos en matemáticas, en comparación a los padres de mujeres, lo que incide sobre su autoconcepto académico (Gladstone et al., 2018) y, en el mismo sentido, el efecto positivo de las predicciones maternales de habilidad sobre la autopercepción y la autoeficacia puede ser más claro en el caso de las hijas (Bleeker \& Jacobs, 2004).

Las percepciones de los padres pueden ser predictivas en función de las tendencias comportamentales previas de los adolescentes. De esta manera, aunque los adolescentes con padres que creen que sus hijos fuman tienen una probabilidad más alta de hacerlo (Mak, 2018), las creencias maternales altas se asocian con el sostenimiento del autoconcepto académico de los hijos solo cuando los adolescentes ya traen alto rendimiento (Pesu et al., 2018). De igual forma, la calibración entre creencias y comportamientos puede estar relacionada con las interacciones previas; así, entre más jóvenes sean los padres y menos reconozcan los problemas de sus hijos, en casos de hijos con más problemas de comportamiento e historiales de tratamiento adolescente y más problemas previos al inicio del tratamiento, puede haber más discrepancia entre la percepción parental y el consumo real de los hijos adolescentes en tratamiento (McGillicuddy \& Eliseo-Arras, 2012).

En cuarto lugar, sobre los valores parentales, la utilidad de la ciencia y la tecnología percibida por los padres influencia el valor asignado por los hijos a estos temas, con la mediación de las percepciones de los hijos sobre los valores de los padres (Šimunović et al., 2018). Aunque los valores percibidos en las madres pueden ser considerados como los que más influyen en los valores de los hijos (Gniewosz \& Noack, 2012a), cuando no hay coincidencia en los valores entre padre y madre, aumenta la probabilidad de que los hijos asignen menos importancia a las áreas académicas (Gniewosz \& Noack, 2012b). En este contexto, después de los rasgos de personalidad de los hijos, los valores parentales pueden influenciar las creencias de los hijos (Bobowik et al., 2011) y su influencia pueden asociarse con otras creencias y con los comportamientos parentales para incidir sobre el nivel de aprendizaje de los hijos (Butler \& Le (2018).

Los valores de los padres sobre la religiosidad pueden asociarse positivamente con el mantenimiento de las creencias religiosas adolescentes aprendidas en el hogar (Webb \& Whitmer, 2003), con el sentido de responsabilidad adolescente a través del estilo autoritativo de crianza (Lindner et al., 1999), y con las creencias de los hijos con la mediación de las percepciones de estos sobre la creencia de sus padres y del deseo propio por compartir las creencias parentales (Okagaki et al., 1999). Las creencias religiosas de las madres y la satisfacción con las relaciones podrían tener más influencia en las hijas que en los hijos varones (Mcneely et al., 2002).

De manera similar, las metas parentales pueden influir en las metas de los hijos (Gonida et al., 2009) y a través de estas incidir en las actitudes adolescentes hacia la búsqueda o evitación de ayuda académica (Gonida, Karabenick, Makara \& Hatzikyriakou, 2014).

En quinto lugar, la autoeficacia parental influye directamente en la autoeficacia adolescente (Di Giunta L., et. Al., 2018), en el ajuste adolescente (Shumow \& Lomax, 2002), en la probabilidad de que los hijos consuman sustancias (Chang, et. Al., 2015), y en las habilidades adolescentes para la realimentación y ganancia de peso en casos de anorexia (Lock et al., 2015). Así mismo, la autoeficacia parental afecta el logro académico adolescente junto con otras creencias, como las expectativas parentales (Chen \& Fish, 2012). No obstante, en la medida que el hijo crece, se disminuye la asociación inversa entre la autoeficacia parental y los comportamientos problemáticos adolescentes (Babskie et al., 2017), disolviéndose la significatividad de esta relación (Sanders et al., 2016).

En un ámbito similar, los padres manifiestan creencias sobre el control de las situaciones, en las cuales el locus de control parental y los comportamientos externalizantes de los hijos puedan ser descritos en una relación bidireccional (Freed \& Tompson, 2011), mientras que las atribuciones parentales sobre el control podrían influir en las dificultades adolescentes para la regulación emocional con la mediación de los comportamientos parentales hostiles (Wang \& Wang, 2018). Así mismo, las creencias de los padres sobre la autoridad legítima pueden ser congruentes con las ideas de sus hijos cuando el control de las situaciones es compartido con ellos (Chen \& Faldowski, 2014).

\section{Discusión}

Las creencias parentales comprometidas en la crianza son construcciones de los padres que operan facilitando la apropiación personal de la experiencia. De acuerdo con lo expuesto, el interés por el estudio de este tema se registra en diferentes regiones geográficas, como se observa en los trabajos analizados, y su relación con otras variables de los padres y de los hijos ha sido confirmada con diferentes tamaños muestrales, lo que reivindica la validez del estudio de las creencias parentales y sus implicaciones (Schaffer, 2006).

Además, los trabajos sobre creencias parentales abordan diferentes tipos de conceptos, lo que hace particular a este campo. Así las creencias generales sobre los atributos y las experiencias humanas abarcan una amplia gama de conceptos no necesariamente 
relacionados con atributos específicos de los hijos, mientras que tanto las expectativas parentales como las percepciones sobre las capacidades de los hijos han sido concebidas como tipos de creencias que vinculan distintos dominios de desempeño para los adolescentes, algo similar a lo que sucede tanto con los valores como con las metas parentales.

Los estudios revisados permiten confirmar que lo que los padres creen sobre sus hijos, sobre sí mismos y sobre los atributos y experiencias humanas puede ser considerado como contribución para dimensionar el rol de la influencia parental dentro del proceso de crianza (Sigel \& McGillicuddy-De Lisi, 2002). En los modelos clásicos, se ha considerado que hay experiencias externas a la relación con los hijos y características propias de los padres relacionadas con el proceso de crianza (Belsky, 1984) y que el sistema parental de creencias es un predictor distal del comportamiento adolescente (Darling \& Steinberg 1993). Más recientemente, el estudio de las creencias parentales ha permitido evaluar las relaciones entre la crianza y las trayectorias en el desarrollo adolescente (Simpkins et al., 2015), proponiéndose un efecto de cascada de las creencias sobre las acciones de los padres, y de estas a su vez sobre las creencias y comportamientos de sus hijos (Bornstein, Putnick \& Suwalsky, 2018).

Con base en la información establecida, hay razones para sugerir que las creencias de los padres son influenciadas por las experiencias y atributos parentales, que inciden en sus actuaciones y que, por esa vía, influyen en las tendencias comportamentales de los hijos. De igual forma, es posible que los atributos de los padres, como su estatus socioeconómico o condición de origen, puedan relacionarse directamente con sus acciones (p. ej. involucrarse en la educación de los hijos), pero a la luz de los trabajos revisados esta relación es más clara con la mediación de creencias parentales como las expectativas (Davis-Kean, 2005).

Adicionalmente, es posible que existan otras fuentes de variación que ayuden a explicar la configuración del sistema de creencias parentales, como los atributos o características propias de los hijos (p. ej.: sexo, estado de salud, capacidad de aprendizaje), y que la relación entre las creencias parentales y el comportamiento de los hijos pueda ser episódica; es decir, los comportamientos previos de los hijos pueden servir de insumo para el origen, la consolidación o el ajuste de las creencias de los padres. De forma complementaria, una manera de comprender las relaciones descritas pasa por establecer que los padres tienden a comportarse en formas compatibles con sus creencias, lo que influye en las inclinaciones cognitivas y comportamentales de sus hijos, planteando la posibilidad de una "profecía autocumplida".

Teniendo en cuenta lo establecido, las creencias parentales pueden ser de tipo general o de dominio específico, de manera que estas últimas pueden ser las que mejor se asocien estadísticamente con las tendencias comportamentales concretas de los padres y de los hijos. Igualmente, frente a un mismo dominio del desarrollo de los hijos puede activarse más de un tipo de creencia parental, como el caso de las expectativas, la percepción de competencia y la autoeficacia en el desempeño académico adolescente, lo que supone una manifestación simultánea como sistema. En todo caso, la influencia de las creencias se ha reportado como sensible al declive en la influencia parental (Babskie et al., 2017), si bien, como configuración incrustada en la relación entre padres e hijos, cabe también la posibilidad de que el sistema de creencias de los padres se transforme en cuanto se alteran los patrones de interacción entre padres e hijos (Pomerantz et al., 2007), lo que resalta la importancia de abordar diseños longitudinales en los estudios dentro del campo.

Dentro de los límites de una revisión, este trabajo reconoce la necesidad de ampliar los planteamientos expuestos con estudios empíricos en diferentes contextos culturales que permitan confirmar o ajustar lo planteado. Así mismo, la exploración de propuestas metodológicas alternativas puede resultar relevante para dimensionar el poder explicativo atribuido al sistema de creencias, como en el caso de las etnoteorías parentales (Harkness \& Super, 1992). De igual forma, futuros estudios pueden expandir su análisis contemplando otras perspectivas vinculadas a las creencias como lo son las cogniciones parentales (Bornstein, 2015). En todo caso, el interés vigente por las creencias parentales resalta sus implicaciones tanto para la investigación como para el ámbito de las intervenciones psicosociales (MoralesCastillo, Aguirre-Dávila \& Durán-Urrea, 2019), dado que el trabajo con padres y adolescentes puede resultar fortalecido.

\section{As crenças parentais na parentalidade e suas relações com o comportamento do adolescente}

Resumo: As crenças parentais são estruturalmente integradas na relação pai-filho, o que pode ser considerado como uma contribuição para entender o desenvolvimento do adolescente. $O$ objetivo deste trabalho é analisar a ligação entre as crenças dos pais e o comportamento dos adolescentes, para o qual realizou-se um levantamento bibliográfico em seis bases de dados, incluindo 53 estudos que avaliaram quantitativamente a influência das crenças dos pais nas práticas parentais com filhos adolescentes. $\mathrm{Na}$ análise das informações, os seguintes conceitos foram destacados como "crenças dos pais": crenças gerais sobre atributos e experiências humanas, expectativas dos pais, percepções das habilidades das crianças, valores e objetivos dos pais, autoeficácia dos pais e crenças sobre o controle de situações. Como conclusões, se estabeleceu que existem indicadores teóricos apropriados para considerar que as crenças parentais são afetadas pelos atributos e experiências dos pais, influenciando em efeito cascata o comportamento das crianças durante a adolescência.

Palavras-chave: crenças parentais, parentalidade, adolescência, práticas parentais. 


\section{Parental beliefs in the parenting process and their relations with adolescent behavior}

Abstract: Parental beliefs are structurally integrated into the relation between parents and adolescents, and could be considered as contributions to understand adolescent development. The objective of this article is to analyze the links between the beliefs of the parents and the behavior of the adolescents, for which a bibliographical review was performed in six databases, including 53 studies that quantitatively measure the influence of the beliefs of the parents in parenting practices with adolescents. In the analysis of the information, the following concepts were highlighted as "parental beliefs": general beliefs about human attributes and experiences, parent expectations, perceptions of the adolescent abilities, parental values and goals, parental self-efficacy and beliefs about control of the situation. As conclusions, it is established that there are appropriate theoretical indicators to consider that parental beliefs are affected by the attributes and experiences of the parents, influencing in a cascade effect the behavior of the adolescents.

Keywords: parental beliefs, parenting, adolescence, parenting practices.

\section{Les croyances parentales dans le processus parental et leurs relations avec le comportement des adolescents}

Résumé: Les croyances des parents sont structurellement intégrées dans la relation parents-enfants et peuvent être considérées comme des contributions à la compréhension du développement des adolescents. L'objectif de cet article est d'analyser les liens entre les croyances parentales et le comportement des adolescents, pour lesquels une revue bibliographique a été développée dans six bases de données, dont 53 études évaluant quantitativement l'influence des croyances parentales sur les pratiques parentales avec les adolescents. Dans l'analyse de l'information, les concepts suivants ont été mis en évidence comme « croyances parentales »: croyances générales sur les attributs et expériences de l'être humain, attentes parentales, perceptions des capacités de l'enfant, valeurs et objectifs parentaux, l'auto-efficacité parentale et les croyances sur le contrôle des situations. En conclusion, il est établi que les attributs et les expériences des parents influent sur les croyances parentales, ce qui a un effet en cascade sur le comportement des adolescents.

Mots-clés: Croyances parentales, Parentalité, Adolescence, Pratiques parentales.

\section{Referencias}

Aguirre-Dávila, E. (2015). Prácticas de crianza, temperamento y comportamiento prosocial de estudiantes de la educación básica. Revista Latinoamericana de Ciencias Sociales: Niñez y Juventud, 13(1), 223-243.

Babskie, E., Powell, D. N., \& Metzger, A. (2017). Variability in parenting self-efficacy across prudential adolescent behaviors. Parenting, 17(4), 242-261. doi: 10.1080/15295192.2017.1369314

Belsky, J. (1984). The determinants of parenting: a process model. Child Development, 55(1), 83-96. doi: $10.2307 / 1129836$

Bleeker, M. M., \& Jacobs, J. E. (2004). Achievement in math and science: Do mothers' beliefs matter 12 years later? Journal of Educational Psychology, 96(1), 97-109. doi: 10.1037/0022-0663.96.1.97

Blum, R. W., Astone, N. M., Decker, M. R., \& Mouli, C. (2014). A conceptual framework for early adolescence: a platform for research. International Journal of Adolescent Medicine \& Health, 26(3), 321-331. doi: 10.1515/ijamh-2013-0327

Bobowik, M., van Oudenhoven, J., Basabe, N., Telletxea, S., \& Páez, D. (2011). What is the better predictor of students' personal values: parents' values or students' personality? International Journal of Intercultural Relations, 35(4), 488-499. doi: 10.1016/j.ijintrel.2011.02.006
Bois, J., Sarrazin, P., Brustad, R., Trouilloud, D., \& Cury, F. (2002). Mothers' expectancies and young adolescents' perceived physical competence: a yearlong study. The Journal of Early Adolescence, 22(4), 384-406. doi: 10.1177/027243102237189

Bornstein, M. H. (2015). Children's parents. In R. M. Lerner, M. H. Bornstein, \& T. Leventhal (Eds.), Handbook of child psychology and developmental science (Vol. 4, pp. 55-132). New Jersey: Wiley.

Bornstein, M., Putnick, D., \& Suwalsky, J. (2018). Parenting cognitions - parenting practices - child adjustment? The standard model. Development and Psychopathology, 1-18. doi: 10.1017/S0954579417000931

Butler, Y., \& Le, V. N. (2018). A longitudinal investigation of parental social-economic status (SES) and young students' learning of English as a foreign language. System, 73, 4-15. doi: 10.1016/j.system.2017.07.005

Calero, F., Dalley, C., Fernandez, N., Davenport-Dalley, T., Morote, E., \& Tatum, S. (2014). A model of academic self-concept for high school Hispanic students in New York. Journal of Latinos and Education, 13(1), 3-43. doi: 10.1080/15348431.2013.800820

Cederbaum, J., Guerrero, E., Barman-Adhikari, A., \& Vincent, C. (2015). Maternal HIV, substance use role modeling, and adolescent girls' alcohol use. The Journal 
of the Association of Nurses in AIDS Care, 26(3), 259-271. doi: 10.1016/j.jana.2014.11.005

Chang, F.-C., Lee, C.-M., Miao, N.-F., Lin, S.-H., Lung, C.-N., Liao, H.-J., . . . Zeng, W.-T. (2015). Parental efficacy and adolescent competence skills associated with adolescent substance use. Journal of Substance Use, 20(2), 85-92. doi: 10.3109/14659891.2013.859752

Chassin, L., Presson, C. C., Rose, J. S., \& Sherman, S. J. (1998). Maternal socialization of adolescent smoking: intergenerational transmission of smoking-related beliefs. Psychology of Addictive Behaviors, 12(3), 206-216. doi: 10.1037/0012-1649.34.6.1189

Chen, Y., \& Faldowski, R. (2014). Beliefs in the legitimacy of decision authority among Chinese adolescents and parents: a person-centered approach. Journal of Adolescence, 37(7), 1121-1133. doi: 10.1016/j.adolescence.2014.07.019

Chen, Y., \& Fish, M. (2012). Parental involvement of mothers with chronic illness and children's academic achievement. Journal of Family Issues, 34(5), 583-606, doi: 10.1177/0192513X12444081

Darling, N., \& Steinberg, (1993). Parenting style as context: an integrative model. Psychological Bulletin, 113(3), 487-496. doi: 10.1037/0033-2909.113.3.487

Davis-Kean, P. E. (2005). The influence of parent education and family income on child achievement: the indirect role of parental expectations and the home environment. Journal of Family Psychology, 19(2), 294-304. doi: 10.1037/0893-3200.19.2.294

Dickens, M. N., \& Cornell, D. G. (1993). Parent influences on the mathematics self-concept of high ability adolescent girls. Journal for the Education of the Gifted, 17(1), 5373. doi: 10.1177/016235329301700106

Dielman, T., Leech, S., Becker, M., Rosenstock, I., Horvath, W., \& Radius, S. (1982). Parental and child health beliefs and behavior. Health Education Quarterly, 9(2-3), 60-77. doi: 10.1177/109019818200900207

Di Giunta L., Iselin A. R., Lansford J. E., Eisenberg N., Lunetti C., Thartori E., . . . Gerbino M. (2018). Parents' and early adolescents' self-efficacy about anger regulation and early adolescents' internalizing and externalizing problems: a longitudinal study in three countries. Journal of Adolescence, 64, 124-135. doi: 10.1016/j.adolescence.2018.01.009

Dweck, C. S. (2012). Implicit theories. In P. A. M. Van Lange, A. W. Kruglanski, \& E. T. Higgins (Eds.), Handbook of theories of social psychology (Vol. 2, pp. 43-61). Thousand Oaks: Sage.

Eng, S. (2012). Cambodian early adolescents' academic achievement: the role of social capital. The Journal of Early Adolescence, 33(3),378-403. doi: 10.1177/0272431612441069

Fishbein, M., \& Ajzen, I. (1975). Belief, attitude, intention, and behavior: an introduction to theory and research. Reading: Addison-Wesley.

Fleming, W. S., Sznajder, K. K., Nepps, M., \& Boktor, S. W. (2018). Barriers and facilitators of HPV vaccination in the VFC Program. Journal of Community Health, 43(3), 448-454. doi: 10.1007/s10900-017-0457-x
Fredricks, J. A., \& Eccles, J. S. (2002). Children's competence and value beliefs from childhood through adolescence: growth trajectories in two male-sex-typed domains. Developmental Psychology, 38(4), 519-533. doi: 10.1037/0012-1649.38.4.519

Freed, R., \& Tompson, M. (2011). Predictors of parental locus of control in mothers of pre- and early adolescents. Journal ofClinical Child \& Adolescent Psychology, 40(1), 100-110. doi: 10.1080/15374416.2011.533410

Gladstone, J. R., Häfner, I., Turci, L., Kneißler, H., \& Muenks, K. (2018). Associations between parents and students' motivational beliefs in mathematics and mathematical performance: the role of gender. Contemporary Educational Psychology, 54, 221-234. doi: 10.1016/j.cedpsych.2018.06.009

Gniewosz, B., \& Noack, P. (2012a). The role of betweenparent values agreement in parent-to-child transmission of academic values. Journal of Adolescence, 35(4), 809-822. doi: 10.1016/j.adolescence.2011.11.007

Gniewosz, B., \& Noack, P. (2012b). What you see is what you get: the role of early adolescents' perceptions in the intergenerational transmission of academic values. Contemporary Educational Psychology, 37(1), 70-80. doi: 10.1016/j.cedpsych.2011.10.002

Gonida, E., Karabenick, S., Makara, K., \& Hatzikyriakou, G. (2014). Perceived parent goals and student goal orientations as predictors of seeking or not seeking help: does age matter? Learning and Instruction, 33, 120-131. doi: 10.1016/j.learninstruc.2014.04.007

Gonida, E., Voulala, K., \& Kiosseoglou, G. (2009). Students' achievement goal orientations and their behavioral and emotional engagement: co-examining the role of perceived school goal structures and parent goals during adolescence. Learning and Individual Differences, 19(1), 53-61. doi: 10.1016/j.lindif.2008.04.002

Hall, M., \& Raimi, K. (2018). Is belief superiority justified by superior knowledge? Journal of Experimental Social Psychology, 76, 290-306. doi: 10.1016/j.jesp.2018.03.001

Halligan, P. (2007). Belief and illness. The Psychologist, 6(2), 358-361

Harkness, S., \& Super, C. (1992). Parental ethnotheories in action. In I. Sigel, A. V. McGillicuddy-De Lisi, \& J. J. Goodnow (Eds.), Parental belief systems: the psychological consequences for children (pp. 373-391). New Jersey: Lawrence Erlbaum Associates.

Heitzler, C., Levin, S., Duke, J., \& Huhman, M. (2006). Correlates of physical activity in a national sample of children aged 9-13 years. Preventive Medicine, 42(4), 254-261. doi: 10.1016/j.ypmed.2006.01.010

Her, P., \& Dunsmore, J. (2011). Parental beliefs about emotions are associated with early adolescents' independent and interdependent self-construals. International Journal of Behavioral Development, 35(4), 317-328. doi: 10.1177/0165025410397644

Hofferth, S., \& Jeong, U. (2016). How do they do it? The immigrant paradox in the transition to adulthood. Social Science Research, 57, 177-195. doi: 10.1016/j. ssresearch.2015.12.013 
Holmes, T., Bond, L., \& Byrne, C. (2008). Mothers' beliefs about knowledge and mother-adolescent conflict. Journal of Social and Personal Relationships, 25(4), 561-586. doi: 10.1177/0265407508090873

Jensen, A. C., \& McHale, S. M. (2015). What makes siblings different? The development of sibling differences in academic achievement and interests. Journal of Family Psychology, 29(3), 469-478. doi: 10.1037/fam0000090

Kimiecik, J., \& Horn, T. (1998). Parental beliefs and children's moderate-to-vigorous physical activity. Research Quarterly for Exercise and Sport, 69(2), 163-175. doi: 10.1080/02701367.1998.10607681

Kirk, C. M., Lewis-Moss, R. K., Nilsen, C., \& Colvin, D. Q. (2011). The role of parent expectations on adolescent educational aspirations. Educational Studies, 37(1), 8999. doi: 10.1080/03055691003728965

Kung, H., \& Lee, C. (2016). Multidimensionality of parental involvement and children's mathematics achievement in Taiwan: Mediating effect of math self-efficacy. Learning and Individual Differences, 47, 266-274. doi: 10.1016/j. lindif.2016.02.004

Lamb, C., \& Crano, W. (2014). Parents' beliefs and children's marijuana use: Evidence for a self-fulfilling prophecy effect. Addictive Behaviors, 39(1), 127-133. doi: 10.1016/j.addbeh.2013.09.009

Leung, J., \& Shek, D. (2014). The influence of parental beliefs on the development of Chinese adolescents experiencing economic disadvantage: maternal control as a mediator. Journal of Family Issues, 37(4), 543-573. doi: 10.1177/0192513X13518776

Leung, J., \& Shek, D. (2018). Family processes and adolescent achievement motivation in poor Chinese single-mother families. Journal of Family Issues, 39(9), 2523-2544. doi: 10.1177/0192513X18757827

Lindner, M., Hetherington, E. M., \& Reiss, D. (1999). Parental religiosity, parenting style, and adolescent social responsibility. The Journal of Early Adolescence, 19(2), 199-225. doi: 10.1177/0272431699019002004

Lock, J., Le Grange, D., Agras, W., Fitzpatrick, K., \& Stainer, M. (2015). Can adaptive treatment improve outcomes in family-based therapy for adolescents with anorexia nervosa? Feasibility and treatment effects of a multi-site treatment study. Behaviour Research and Therapy, 73, 90-96. doi: 10.1016/j.brat.2015.07.015

Mak, H. W. (2018). Parental belief and adolescent smoking and drinking behaviors: a propensity score matching study. Addictive Behaviors Reports, 8, 11-20. doi: 10.1016/j.abrep.2018.04.003

Malmberg, L. E., Ehrman, J., \& Lithén, T. (2005). Adolescents' and parents' future beliefs. Journal of Adolescence, 28(6), 709-723. doi: 10.1016/j. adolescence.2004.12.007

McDonald, N., Deakin, E., \& Aalborg, A. (2010). Influence of the social environment on children's school travel. Preventive Medicine, 50, s65-s69. doi: 10.1016/j. ypmed.2009.08.016
McGillicuddy, N., \& Eliseo-Arras, R. (2012). Parentadolescent report correspondence on adolescent substance abuse among teens in residential rehabilitation. Addictive Behaviors, 37(4), 456-462. doi: 10.1016/j. addbeh.2011.12.006

McNeely, C., Shew, M., Beuhring, T., Sieving, R., \& Blum, R. (2002). Mothers' influence on the timing of first sex among 14- and 15-year-olds. Journal of Adolescent Health, 31(3), 256-266. doi: 10.1016/S1054139X(02)00350-6

Mesurado, B., Richaud, M., Mestre, M., Samper-García, P., Tur-Porcar, A., Morales, S., \& Viveros, E. (2014). Parental expectations and prosocial behavior of adolescents from low-income backgrounds: a cross-cultural comparison between three countries - Argentina, Colombia, and Spain. Journal of Cross-Cultural Psychology, 45(9), 1471-1488. doi: 10.1177/0022022114542284

Morales-Castillo, M., \& Aguirre-Dávila, E. (2018). Involucramiento parental basado en el hogar y logro académico en la adolescencia. Revista Colombiana de Psicología, 27(2), 137-160. doi: 10.15446/rcp.v27n2.66212

Morales-Castillo, M., Aguirre-Dávila, E., \& Durán-Urrea, L. (2019). Los contenidos de la formación parental y sus implicaciones para el comportamiento de los adolescentes: elementos desde una revisión. Saúde e Sociedade, 28(3), 224-238. doi: 10.1590/s010412902019181111

Okagaki, L., Hammond, K., \& Seamon, L. (1999). Socialization of religious beliefs. Journal of Applied Developmental Psychology, 20(2), 273-295. doi: 10.1016/S0193-3973(99)00017-9

Pesu, L., Aunola, K., Viljaranta, J., \& Hirvonen, R. (2018). The role of mothers' beliefs in students' self-concept of ability development. Learning and Individual Differences, 65, 230-240. doi: 10.1016/j. lindif.2018.05.013

Pomerantz, E., Moorman, E., \& Litwack, S. (2007). The how, whom, and why of parents' involvement in children's academic lives: more is not always better. Review of Educational Research, 77(3), 373-401. doi:10.3102/003465430305567

Power, T. G. (2013). Parenting dimensions and styles: a brief history and recommendations for future research. Childhood Obesity, 9(1), 14-21. doi: 10.1089/ chi.2013.0034

Sanders, W., Parent, J., Forehand, R., Sullivan, A., \& Jones, D. (2016). Parental perceptions of technology and technologyfocused parenting: associations with youth screen time. Journal of Applied Developmental Psychology, 44, 28-39. doi: 10.1016/j.appdev.2016.02.005

Schaffer, H. R. (2006). Parental belief systems. In H. R. Schaffer, Key concepts in developmental psychology (pp. 184-188). London: Sage

Sharot, T., \& Garrett, N. (2016). Forming beliefs: why valence matters. Trends in Cognitive Sciences, 20(1), 25-33. doi: 10.1016/j.tics.2015.11.0022 
Shumow, L., \& Lomax, R. (2002). Parental efficacy: predictor of parenting behavior and adolescent outcomes. Parenting, 2(2), 127-150. doi: 10.1207/S15327922PAR0202_03

Sigel, I., \& McGillicuddy-De Lisi, A. (2002). Parent beliefs are cognitions: the dynamic belief systems model. In M. Bornstein (Ed.), Handbook of parenting (Vol. 3, pp. 485-508). New Jersey: Lawrence Erlbaum Associates.

Sigel, I., McGillicuddy-De Lisi, A., \& Goodnow, J. J. (1992). Parental belief systems: the psychological consequences for children. New Jersey: Lawrence Erlbaum Associates.

Simpkins, S. D., Fredricks, J. A., \& Eccles, J. S. (2012). Charting the Eccles' expectancy-value model from mothers' beliefs in childhood to youths' activities in adolescence. Developmental Psychology, 48(4), 1019-1032. doi: 10.1037/a0027468

Simpkins, S. D., Fredricks, J. A., \& Eccles, J. S. (2015). The role of parents in the ontogeny of achievement-related motivation and behavioral choices. Monographs of the Society for Research in Child Development, 80(2). doi: 10.1111/mono.12160

Šimunović, M., Reić Ercegovac, I., \& Burušić, J. (2018). How important is it to my parents? Transmission of STEM academic values: the role of parents' values and practices and children's perceptions of parental influences. International Journal of Science Education, 40(9), 977-995. doi: 10.1080/09500693.2018.1460696
Sorkkila, M., Aunola,K., \& Ryba, T.(2017). A person-oriented approach to sport and school burnout in adolescent student-athletes: the role of individual and parental expectations. Psychology of Sport and Exercise, 28, 58-68. doi: 10.1016/j.psychsport.2016.10.004

Valois, M., Rizzo, J., Wanick, S., Cavalcanti, E., \& Assis, F. (2012). Effect of exercise-induced bronchospasm and parental beliefs on physical activity of asthmatic adolescents from a tropical region. Annals of Allergy, Asthma \& Immunology, 108(4), 249-253. doi: 10.1016/j. anai.2012.01.016

Wang, M., \& Wang, J. (2018). Negative parental attribution and emotional dysregulation in Chinese early adolescents: harsh fathering and harsh mothering as potential mediators. Child Abuse \& Neglect, 81, 12-20. doi: 10.1016/j.chiabu.2018.04.008

Webb, M., \& Whitmer, K. (2003). Parental religiosity, abuse history and maintenance of beliefs taught in the family. Mental Health, Religion \& Culture, 6(3), 229-239. doi: 10.1080/1367467021000038156

Recibido: $18 / 05 / 2020$

R1: $03 / 10 / 2019$

R2: $21 / 02 / 2020$

Aprobado: 09/04/2020 